\title{
Ovarian Cancer by AJCC v8 Stage
}

National Cancer Institute

\section{Source}

National Cancer Institute. Ovarian Cancer by AJCC v8 Stage. NCI Thesaurus. Code C139963.

A term that refers to the staging of ovarian cancer according to the American Joint Committee on Cancer, 8th edition. This stag ing system applies to ovarian carcinomas. Nonepithelial primary ovarian cancers may be staged using this classification but should be reported separately. (from AJCC 8th Ed.) 